\title{
Tumor endometrioide borderline de ovário em adolescente: relato de caso
}

Primeira submissão em 12/01/10 Última submissão em 13/01/10 Aceito para publicação em 07/02/10 Publicado em 20/04/10

\section{Borderline ovarian endometrioid tumor in adolescent: case report and review of the literature}

\author{
Tamara Carvalho dos Santos'; Paulo Zanvettor²; Iguaracyra Barreto de Oliveira Araújo³
}

\section{unitermos \\ Tumor ovariano em \\ adolescente \\ Tumor borderline \\ do ovário \\ Tumor endometrioide \\ do ovário}

\section{resumo}

Os tumores endometrioides malignos ovarianos são neoplasias epiteliais raras em pacientes jovens. Enquanto os carcinomas endometrioides ovarianos acometem mulheres na quinta e na sexta décadas de vida, os tumores borderline acometem mulheres em uma faixa etária mais ampla (de 22 a 77 anos). Descreveremos a seguir um caso raro de tumor endometrioide borderline ocorrendo em paciente de 16 anos. Desde que tumores do saco vitelino (TSV) e de Sertoli-Leydig (TCSL) podem mimetizar morfologicamente tumor endometrioide, o uso da imuno-histoquímica, além dos aspectos morfológicos, foi fundamental para estabelecer o diagnóstico diferencial entre essas condições.

\section{abstract}

Malignant endometrioid ovarian tumors are rare epithelial neoplasias among young patients. Whereas endometrioid ovarian carcinomas are more common among women in their fifth and sixth decades of life, borderline endometrioid tumors affect 22 to 77 year-old women. The present study reports a rare case of borderline endometrioid tumor in a 16 year-old patient. Since yolk sac and Sertoli-Leydig cell tumor may morphologically mimic endometrioid tumor, both the use of immunohistochemistry and morphological aspects were essential to establish the differential diagnosis. key words

Ovarian tumor in adolescent

Borderline ovarian tumor

Endometrioid ovarian tumor

1. Graduanda em Medicina pela Faculdade de Medicina da Bahia da Universidade Federal da Bahia (FMB-UFBA); bolsista de iniciação cientifica do Conselho Nacional de Desenvolvimento Científico e Tecnológico (CNPq).

2. Doutora; professora adjunta de Patologia da FMB-UFBA; pesquisadora do Hospital Aristides Maltez (HAM).

3. Cirurgião oncológico do HAM. 


\section{Introdução}

A frequência dos diferentes subtipos histológicos dos tumores ovarianos varia de acordo com a idade ${ }^{(14,25)}$. Enquanto na população abaixo dos 19 anos predominam os tumores de células germinativas (TCG) ${ }^{(18,21,24,25)}$, em mulheres adultas predominam os de origem epitelial ${ }^{(14)}$. Entre os tumores epiteliais malignos ocorrendo em mulheres, cerca de $10,6 \%$ correspondem aos carcinomas endometrioides ${ }^{(14)}$. Em crianças e adolescentes, os tumores epiteliais correspondem a $30 \%$ dos tumores ovarianos ${ }^{(24)}$. Entre os malignos, a proporção dos tumores epiteliais borderline em jovens e crianças varia de $16 \%$ a $84 \%{ }^{(15,22,24)}$, predominando o subtipo seroso, seguido pelo mucinoso ${ }^{(22)}$. Porém, tumores endometrioides malignos ou borderline não têm sido descritos nessa faixa etária ${ }^{(1,15,18,21,22,24,25)}$.

Os tumores endometrioides de ovário assemelham-se morfologicamente às neoplasias endometrioides uterinas e, a exemplo de outros tumores epiteliais, podem ser classificados em benignos, malignos e limítrofes (borderline) $^{(8,10)}$. Em mulheres adultas, os malignos correspondem de $10 \%$ a $25 \%$ de todos os tumores de ovário, sendo que $3 \%$ a $18 \%$ desses carcinomas são de baixo potencial maligno ou borderline ${ }^{(10)}$.

O carcinoma endometrioide ocorre com mais frequência em mulheres entre 50 e 60 anos $^{(10)}$, com relato de um caso abaixo dos 21 anos de idade ${ }^{(22)}$. Esses carcinomas apresentam-se, na maioria das vezes, como um tumor sólidocístico, bilateral em $28 \%$ dos casos. Clinicamente, costuma ser assintomático, apresentando-se como uma massa pélvica, dolorosa ou não, com elevação sérica de cancer antigen (CA) 125 em $80 \%$ dos casos. Morfologicamente, são caracterizados por presença de estruturas glândulopapilares atípicas e confluentes, elevada atividade mitótica e invasão estromal(2). Mórulas de células escamosas bem diferenciadas podem ser observadas em $30 \%$ a $50 \%$ dos $\operatorname{casos}^{(10,23)}$. Luteinização de células estromais não neoplásicas pode ser vista em 10\% dos casos. Os carcinomas endometrioides de ovário estão associados em $15 \%$ a $20 \%$ dos casos a carcinomas endometrioides de outras regiões e, em cerca de $15 \%$ a $42 \%$, ocorre concomitantemente a endometriose $\mathrm{e}^{(4,8,23)}$. Ainda assim, a maioria desses tumores é considerada originária da própria superfície epitelial do ovário.

O tumor endometrioide borderline é uma neoplasia rara que acomete pacientes entre 22 e 77 anos, embora Karen Bell et al. tenha relatado, em sua série de casos, uma faixa etária mais ampla, de 24 a 85 anos $^{(2)}$. Clinicamente, apresenta-se como uma massa pélvica palpável unilateral ou com sangramento uterino. Do ponto de vista morfológico, é caracterizado pela Organização Mundial da Saúde (OMS) como um tumor composto por glândulas endometrioides atípicas ou citologicamente malignas, porém sem invasão do estroma ${ }^{(10)}$. Mais comumente é sólido-cístico, sendo a região sólida caracterizada pela presença de glândulas com atipia citológica em meio a estroma fibromatoso e a região cística, pelo conteúdo hemorrágico ou mucoso(2). Atividade mitótica é usualmente baixa ${ }^{(10)}$, metaplasia escamosa é comum e áreas com hemorragia, infarto e necrose podem existir $\left.{ }^{2}, 10\right)$.

O prognóstico dos tumores endometrioides malignos está relacionado com o estadiamento e o grau histológico, sendo que o tumor borderline exibe excelente prognóstico quando comparado ao carcinoma endometrioide ${ }^{(10)}$. Considerando a ocorrência ímpar de tumor endometrioide borderline de ovário em adolescente, relatamos um caso raro ocorrendo em uma paciente de 16 anos. Esse caso ilustra a necessidade do uso de imuno-histoquímica para estabelecer diagnóstico diferencial com tumores próprios dessa idade, que podem exibir padrão endometrioide, como tumor do saco vitelino (TSV) e tumor de células de Sertoli-Leydig

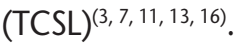

\section{Relato de caso}

Paciente A.V.S., 16 anos, virgem, queixa-se de amenorreia há um ano e tumoração não dolorosa na fossa ilíaca direita há seis meses. Perda ponderal de $3 \mathrm{~kg}$. Ao exame ginecológico, observou-se massa palpável, endurecida, móvel e indolor em região hipogástrica com cerca de $10 \mathrm{~cm}$. Tomografia computadorizada da pelve revelou formação ovoide de contornos parcialmente definidos e densidade heterogênea, composta por áreas sólidas e císticas, localizada na pequena pelve à direita. Ultrassonografia pélvica indicou provável tumor ovariano. Ao exame laboratorial, antígeno carcinoembrionário (CEA) normal, níveis séricos elevados de CA 125 (71,7 U/ml) e de sulfato de deidroepiandrosterona (SDHEA)(346 ug/dl). Foi realizada salpingo-ooforectomia direita.

O estudo histopatológico identificou tumoração ovariana íntegra, de superfície lisa, medindo $13 \times 8 \times 8 \mathrm{~cm}$, predominantemente sólida, com áreas císticas contendo material mucoide acastanhado. A trompa não apresentou alteração. À microscopia, foi observada neoplasia constituída por glândulas e túbulos com moderadas atipias nucleares e frequentes mitoses em meio a estroma proliferado luteinizado (Figura 1), mórulas de células escamosas bem diferenciadas sem atipias (Figura 2), cavidades revestidas por epitélio 
secretor ciliado tipo tubário, com formação de papilas sem atipias (Figura 3) e cápsula ovariana preservada.

Para auxiliar no diagnóstico diferencial com tumores ovarianos comuns nessa faixa etária, foi utilizada a imuno-histoquímica (método LSAB-Dako). A recuperação antigênica foi realizada em calor úmido usando tampão citrato $0,1 \mathrm{M}$ e pH 6 . As células neoplásicas expressaram fortemente pancitoqueratina, citoqueratina 7 (Figura 4), antígeno da membrana epitelial (EMA), receptores de

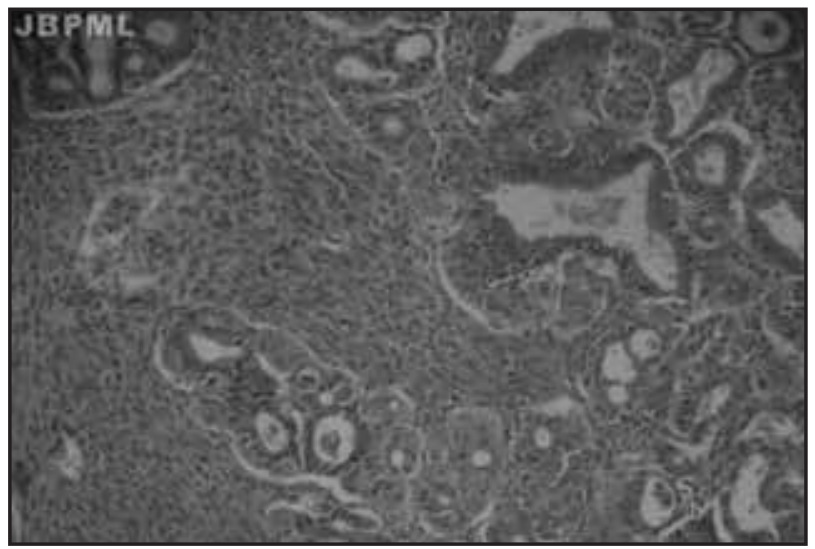

Figura 1 - Aspecto morfológicos (hematoxilina e eosinaimagem 1): padrões glandula e tubular em meio a estroma celuloso luteinizado (200x)

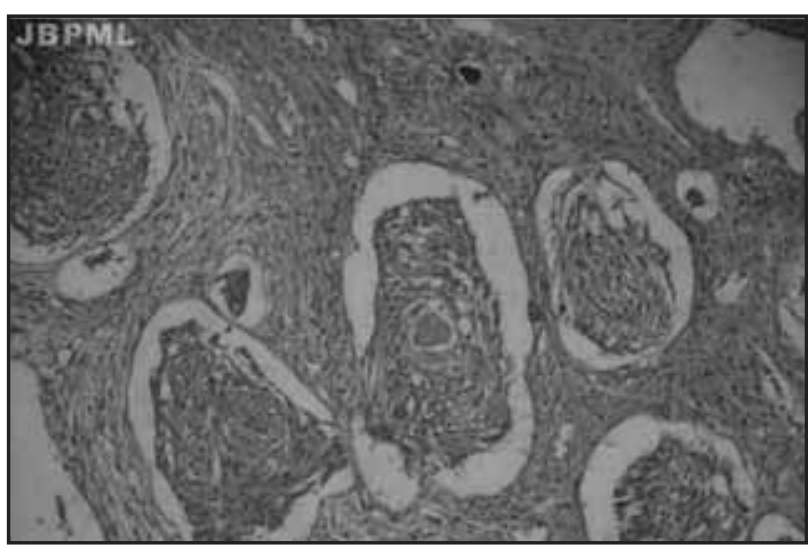

Figura 2 - Aspectos morfológicos (hematoxilina e eosinaimagem 1): diferenciação escamosa com formação de mórulas (300x)

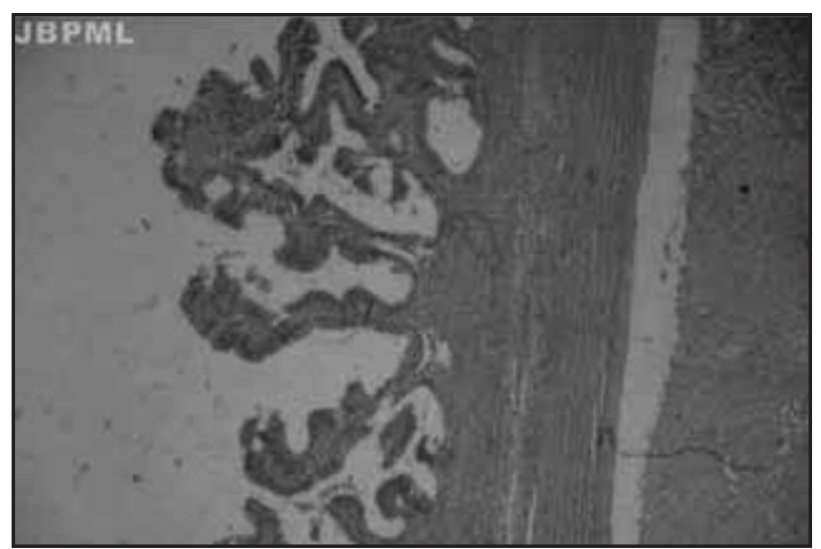

Figura 3 - Aspectos morfológicos (hematoxilina e eosinaimagem 1): áreas císticas com formação de papilas (250x) estrógeno e progesterona. As células foram negativas para proteína placentária alcalina (PLAP), citoqueratina 20, inibina e $\alpha$-fetoproteína. Foi estabelecido o diagnóstico de tumor endometrioide borderline de comportamento imprevisível devido ao elevado índice mitótico (6 mitoses/10 campos de grande aumento).

Cerca de cinco meses após a cirurgia, a menstruação retornou ao padrão regular anterior e os exames laboratoriais e de imagem não mostraram anormalidade. Atualmente, 44 meses após a cirurgia, a paciente encontra-se completamente assintomática.

\section{Discussão}

Os tumores ovarianos malignos em crianças e adolescentes diferem significativamente quanto ao tipo histológico quando comparado aos em adultos ${ }^{(14,25)}$. Enquanto os tumores epiteliais são mais frequentes em adultos, os TCGs predominam em crianças e adolescentes. Entre 15 e 19 anos, os carcinomas correspondem a cerca de $17,7 \%$, sendo que a proporção dos de baixo grau de malignidade ou borderline tem grande variabilidade nas diversas séries pediátricas ${ }^{(15,22,24)}$. Tanto tumores epiteliais serosos quanto mucinosos malignos têm sido descritos no grupo pediátrico abaixo dos 19 anos $^{(1,22)}$. Carcinomas endometrioides de baixo potencial de malignidade e invasores têm sido referidos como raros antes dos 20 anos ${ }^{(22)}$. Devido aos critérios histopatológicos distintos aplicados ao longo do tempo e à abrangência de faixas etárias distintas, há dificuldade em comparar os estudos existentes acerca da frequência dos tumores epiteliais borderline ovarianos em adolescentes. Porém nas diversas casuísticas não há, do nosso conhecimento, qualquer caso de tumor endometrioide borderline descrito antes dos 19 anos (Tabela 1) $)^{(1,15,18,21,22,24,25)}$.

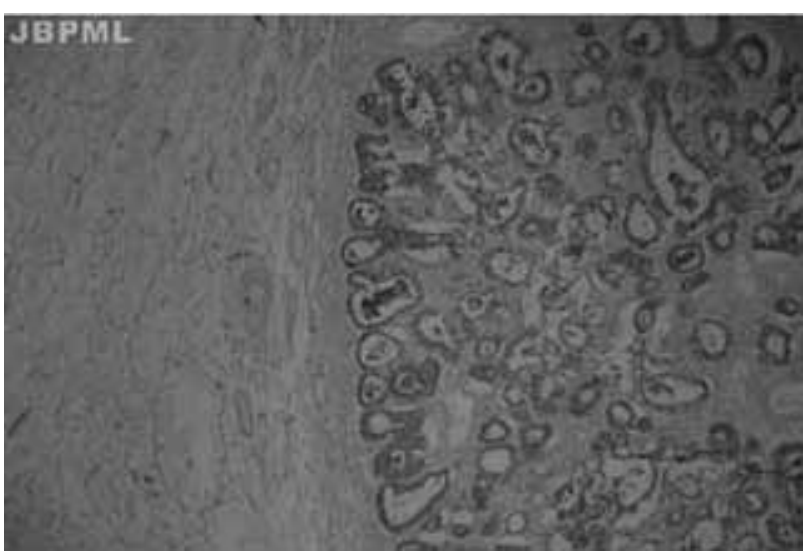

Figura 4 - Aspectos imunofenotípicos (streptoavidina-biotina-peroxidaseimagem 1): expressão de citoceratina 7 em túbulos neoplásicos 


\section{Tabela 1 Tumores epiteliais malignos invasores e borderline de ovário em crianças, adolescentes e adultos jovens}

\begin{tabular}{|c|c|c|c|c|c|}
\hline Estudos & $\begin{array}{l}\text { LMP/total de } \\
\text { tumores epiteliais }\end{array}$ & $\begin{array}{l}\text { Idade } \\
\text { média }\end{array}$ & $\begin{array}{c}\text { LMP } \\
\text { mucinoso }\end{array}$ & $\begin{array}{l}\text { LMP } \\
\text { seroso }\end{array}$ & $\begin{array}{l}\text { Malignos } \\
\text { invasores }\end{array}$ \\
\hline Piippo et al., 1999 (< 17 anos) & $01-/ 17 * *$ & - & 1 & - & 3 \\
\hline Tempelman et al., 2000 (<21 anos) & 0-/10 malignos & - & - & - & 0 \\
\hline Tsal et al., 2001 (<19 anos)* & $6 / 8$ malignos & $17,45 \pm 2$ & 2 & 4 & 2 \\
\hline Morowitz et al., 2003 (<18 anos) & $7-/ 19 * *$ & - & & 4 & 3 \\
\hline Young et al., 2003 (15-19 anos)* & $\begin{array}{l}\text { 122-/179 } \\
\text { malignos }\end{array}$ & - & - & - & 57 \\
\hline You et al., 2005 (<25 anos) & $24-/ 35^{* *}$ & & & & 0 \\
\hline Aggarwal et al., 2007 (<19 anos) & $5 / 25^{* *}$ & $14,4 \pm 2,4$ & 3 & 2 & 0 \\
\hline
\end{tabular}

LMP: tumor epitelial de baixo potencial de malignidade ou borderline (limitrofe).

${ }^{*}$ Representados apenas os casos no intervalo de idade referido. ${ }^{* *}$ Todas as neoplasias tumores epiteliais benignas e malignas.

Os tumores endometrioides possuem, em geral, um padrão histológico bem definido, porém em uma paciente tão jovem impõe-se o diagnóstico diferencial com outras neoplasias ovarianas, que podem exibir aspecto morfológico endometrioide, como TCSLs e TSVs ${ }^{(13,16,19)}$.

Os tumores do cordão sexual correspondem a $4 \%$ dos tumores ovarianos em pacientes de 15 a 19 anos $^{(25)}$, porém não há dados especificamente acerca da frequência do TCSL nesse grupo etário. Por outro lado, segundo Templeman et al., esse foi o segundo tumor ovariano maligno mais frequente abaixo dos 21 anos, superado apenas pelos TCGs. Em geral, esses tumores são raros e acometem unilateralmente mulheres jovens ${ }^{(16)}$, porém podem ocorrer em uma faixa etária mais ampla, de 2 a $76 \operatorname{anos}^{(3,16)}$. Do ponto de vista clínico, esses tumores podem estar associados a anormalidades menstruais, porém, elevação do CA 125, como observada no caso da paciente descrito anteriormente, é muito rara ${ }^{(3)}$. Semelhante aos tumores endometrioides, os TCSLs podem apresentar aspecto macroscópico sólido, com áreas císticas e arranjos tubular e alveolar à microscopia. Entretanto, mesmo nos casos com aspecto endometrioide, a presença de mórulas de células escamosas não são observadas $^{(16)}$. O perfil imuno-histoquímico dos TCSLs é nitidamente distinto dos tumores endometrioides, caracterizado pela positividade para calretinina e inibina e negatividade para EMA (Tabela 2) $)^{(5,6,9,11,12,16,17,20,26)}$. Assim, os aspectos clínicos, morfológicos e imunofenotípicos desfavoreceram o diagnóstico de TCSL, variante endometrioide.

Os TCGs correspondem a aproximadamente $54 \%$ dos tumores malignos ovarianos entre 15 e 19 anos, sendo que o TSV corresponde a $16,3 \%$ desses $^{(25)}$.
TSVs acometem frequentemente adolescentes, porém não costumam apresentar-se com amenorreia. Semelhante aos tumores endometrioides, podem mostrar arquitetura glandular e áreas de luteinização. Por outro lado, áreas de diferenciação escamosa em meio ao estroma não são descritas em TSVs. Contribuindo para distinção entre essas entidades, o perfil imunofenotípico desses tumores é caracterizado pela positividade para $\alpha$-fetoproteína e PLAP. Adicionalmente, enquanto tais tumores são negativos para citoqueratina 7 e EMA, os tumores endometrioides são positivos (Tabela 2$)^{(7,19)}$. Novamente o caso relatado possui características morfológicas (presença de mórulas de células escamosas) e imuno-histoquímicas (positividade para citoqueratina 7 e EMA e negatividade para $\alpha$-fetoproteína e PLAP) que permitem afastar o diagnóstico de TSV com áreas endometrioides.

Outra dificuldade no caso relatado foi estabelecer o diagnóstico diferencial entre adenocarcinoma endometrioide e tumor endometrioide borderline. Considerando que ambas as entidades apresentam o mesmo perfil imuno-histoquímico e manifestações clínicas, o diagnóstico diferencial foi baseado exclusivamente em critérios morfológicos. A OMS considera a invasão estromal o critério mais importante para tal diagnóstico, sendo que a ausência de invasão estromal caracteriza a variante borderline, embora não defina claramente os parâmetros de invasão. Isso é questionado por Bell e Kurman(2), que apontam a reatividade estromal como uma evidência inválida de invasão, pois os tumores endometrioides, tanto borderline quanto malignos francos, costumam apresentar um denso estroma fibroso, simulando reati- 
Aspectos imuno-histoquímicos dos

tumores endometrioides borderline de

ovário em comparação a outros tumores

Tabela 2 ovarianos

$\begin{array}{lccccc} & \text { EMA } & \text { Inibina } & \text { CK 7 } & \text { AFP } & \text { PLAP } \\ \text { TEO } & + & - & + & - & - \\ \text { TCSL } & - & + & + & - & - \\ \text { TSV } & - & - & - & + & +\end{array}$

EMA: Antígeno da membrana epitelial; AFP: alfa-fetoproteína; PLAP: proteína placentária alcalina; TEO: tumor endometrioide de ovário; TCSL: tumor de célula de Sertoli-Leydig; TSV: tumor de saco vitelino; -: negativo; + positivo.

vidade do estroma. Assim, tais autores propõem outros dois critérios de invasão: padrão glandular cribriforme e arquitetura glandular confluente. $\mathrm{O}$ caso relatado apresentava áreas cribriformes, porém a arquitetura não era confluente. Dessa forma, segundo Bell e Kurman, esse tumor seria classificado como endometrioide proliferativo atípico com grau 3 de atipia nuclear, devido ao elevado índice mitótico. A paciente permanece sem queixas 44 meses após a ooforectomia.

\section{Conclusão}

Esse caso ilustra a necessidade de métodos complementares de imuno-histoquímica para estabelecer o diagnóstico diferencial de tumores ovarianos, principalmente quando da sua ocorrência em faixa etária inesperada. Diante das peculiaridades do estroma reativo próprio dos tumores endometrioides, houve dificuldade em analisar os critérios de invasão apresentados pela $\mathrm{OMS}^{(10)}$. Com relação aos critérios de invasão definidos por Bell e Kurman, esse caso preencheu um dos dois critérios. Entretanto, considerando os critérios da OMS, esse tumor foi classificado como endometrioide borderline do ovário. Embora muito raro em adolescente, o perfil imuno-histoquímico aliado a aspectos morfológicos permitiram estabelecer esse diagnóstico. Porém, os critérios de invasão estromal precisam de melhor definição nesses tumores para que parâmetros mais seguros possam ser adotados.

\section{Referências}

1. AGGARWAL, A. et al. Ovarian epithelial tumors of low malignant potential: a case series of 5 adolescent patients. J Pediatric Surg, v. 44, p. 2023-7, 2009.

2. BELL, K. A.; KURMAN, R. J. A clinicopathologic analysis of atypical proliferative (borderline) tumors and welldifferentiated endometrioid adenocarcinomas of the ovary. Am J Surgical Pathol, v. 24, n. 11, p. 1465-79, 2000.

3. CARINGELLA, A. et al. A case of Sertoli-Leydig cell tumor in postmenopausal woman. Int J Gynecol Can, v. 16, p. 435-8, 2006.

4. CASTIBLANCO, A. et al. Rol patogênico del gen supresor de tumores PTEN en cáncer ovárica asociado a endometriosis. Rev Med Chil, v. 134, p. 271-8, 2006.

5. DEAVERS, M. T. et al. Ovarian sterois cell tumors: an immunohistochemical study including a comparison of calretinin with inhibin. Int J Gynecol Pathol, v. 22, p. 162-7, 2003.

6. DEAVERS, M. T. et al. Ovarian sex cord-stromal tumors: an immunohistochemical study including a comparison of calretinin and inhibin. Mod Pathol, v. 16, p. 584-90, 2003.

7. DEVOUASSOUX-SHISHEBORAN, M.; SCHAMMEL, D. P.; TAVASSOLI, F. A. Ovarian hepatoid yolk sac tumors: morphological, immunohistochemical and ultrastructural features. Histopathology, v. 34, p. 462-9, 1999.
8. GILKS, C. B. Subclassification of ovarian surface epithelial tumors based on correlation of histologic and molecular pathologic data. Int J Gynecol Pathol, n. 23, p. 200-5, 2006.

9. KOMMOSS, F. et al. Inhibin immunohistochemical study. Mod Pathol, v. 11, p. 656-64, 1998.

10. LEE, K. R. et al. Tumours of the ovary and peritoneum. In: TAVASSOLI, F. A.; DEVILEE, P. Pathology \& genetics tumors of the breast and female genital organs. WHO Classification of Tumours. Lyon, França: IARC Press, 2003. cap. 2, p. 113-96.

11. MATIAS-GUIU, X.; PONS, C.; PRAT, I. Mullerian inhibiting substance, alphai-nhibin, and CD99 expression in sex cord-stromal tumors and endometrioid ovarian carcinomas resembling sex cord-stromal tumors. Hum Pathol, v. 29, p. 840-45, 1998.

12. MCCLUGGAGE, W. G. Value of inhibin staining in gynecological pathology. Int J Gynecol Pathol, v. 20, p. 70-85, 2001.

13. MCCLUGGAGE, W. G; YOUNG, R. H. Ovarian SertoliLeydig cell tumors with pseudoendometriod tubules (pseudoendometrioid Sertoli-Leydig cell tumors). Am J Surg Pathol, v. 31, p. 592-7, 2007.

14. MINK, P. J.; SHERMAN, M. E.; DEVESA, S. S. Incidence patterns of invasive and borderline ovarian tumors among white women and black women in the united states. Results from the SEER program, 1978-1998. Cancer, v. 95, n. 11, p. 2380-9, 2002. 
15. MOROWITZ, M.; HUFF, D.; ALLMEN, D. Epithelial ovarian tumors in children. J Pediatric Surg, v. 38, n. 3, p. 331-5, 2003.

16. OLIVA, E.; ALVAREZ, T.; YOUNG, R. H. Sertoli cell tumors of the ovary: a clinicopathologic and immunohistochemical study of 54 cases. Am J Surg Pathol, v. 29, n. 2, p. 143-56, 2005.

17. PELKEY, T. J. et al. The diagnostic utility of inhibin staining in ovarian neoplasms. Int J Gynecol Pathol, v. 17, p. 97-105, 1998.

18. PIIPPO, S. et al. Surgery for ovarian masses during childhood and adolescence: a report of 79 cases. J Pediatr Adolesc Gynecol, v. 12, p. 223-7, 1999.

19. RAMALINGAM, P. et al. The use of cytokeratin 7 and EMA in the differentiating ovarian yolk sac tumors from endometrioid and clear cell carcinomas. Am J Surgical Pathol, v. 28, p. 1499-505, 2004.

20. RIOPEL, M. A. et al. Inhibin and epithelial membran antigen immunohistochemistry assist in the diagnosis of sex cord-stromal tumors and provide clues to the histogenesis of hypercalcemic small cell carcinomas. Int J Gynecol Pathol, v. 17, p. 46-53, 1998.
21. TEMPLEMAN, C. et al. Noninflamatory ovarian masses in girls and young women. Obstetrics and Gynecol, v. 96, n. 2, p. 229-33, 2000.

22. TSAL, J. Y. et al. Diagnosis, pathology, stagin, treatment, and outcome of epithelial ovarian neoplasia in patients age $<21$ years. Cancer, v. 91, p. 2065-70, 2001.

23. WU, T. I. et al. Ovarian edometrioid carcinoma with diffuse pigmented peritoneal keratin granulomas: a case report and review of the literature. Int J Gynecol Cancer, v. 16, p. 426-9, 2006.

24. YOU, W. et al. Gynecologic malignancies in women aged less than 25 years. Obstetric and Gynecol, v. 105, n. 6, p. 1405-9, 2005.

25. YOUNG Jr, J. L. et al. Ovarian cancer in children and young adults in the United States, 1992-1997. Cancer, v. 97, p. 2696-700, 2003.

26. ZHEN, W.; SENTURK, B.; PARKASH, V. Inhibin immunohistochemical staining: a practical approach for the surgical pathologist in the diagnoses of ovarian sex cord-stromal tumors. Adv Ant Pathol, v. 10, p. 27-38, 2003. 